\title{
Extirpation of a Ruptured Anterior Spinal Artery Aneurysm Accompanied by Dural Arteriovenous Fistula at the Craniovertebral Junction via a Posterolateral Approach: The Management of Extradural Venous Congestion
}

\author{
Shunya Hanakita ${ }^{1}$ Soichi Oya ${ }^{1}$ Tsukasa Tsuchiya ${ }^{1}$ Masaaki Shojima ${ }^{1}$ Toru Matsui ${ }^{1}$ \\ ${ }^{1}$ Department of Neurosurgery, Saitama Medical Center/University, \\ Saitama Japan \\ Address for correspondence Soichi Oya, MD, PhD, Department of \\ Neurosurgery, Saitama Medical Center/University, 1981 Kamoda, \\ Kawagoe, Saitama 350-8550, Japan (e-mail: sooya-tky@umin.ac.jp).
}

J Neurol Surg B 2019;80(suppl S4):S344-S345.

\begin{abstract}
Objective This study was aimed to discuss how to control extradural venous congestion with an increased pressure in cases of arteriovenous shunt disease of the craniovertebral junction.

Design The study is presented through an operative video.

Results A 77-year-old patient with subarachnoid hemorrhage had a dural arteriovenous fistula located at the C1-C2 level. Left vertebral angiography showed a fistula between the left C2 radiculomeningeal muscular artery and perivertebral plexus. Furthermore, right vertebral angiography showed a ruptured aneurysm at the aberrant branch of the anterior spinal artery originating from the contralateral vertebral artery (VA), possibly formed because of the concurrently increased pressure of the perimedullary veins. Aneurysm extirpation was planned through a posterolateral approach. To reduce venous bleeding during the approach, preoperative embolization of the radiculomeningeal muscular artery was performed. During surgery, the suboccipital triangle was exposed following layer-bylayer dissection of the suboccipital muscles (-Figs. 1 and 2). Subperiosteal dissection of the paravertebral plexus surrounding the $V A$ around the $C 1$ lamina was effective to avoid venous

\section{Keywords}

- anterior spinal artery

- craniovertebral junction

- dural arteriovenous fistula

- surgery bleeding. A bloodless operative field was achieved, and key anatomical structures, such as the $\mathrm{C} 2$ nerve root, feeder, and V3 portion of the left VA, were clearly identified. With a sufficient amount of lateral exposure, the ruptured anterior spinal artery aneurysm was successfully extirpated with bipolar coagulation. The patient was discharged with no neurologic deficit. Conclusion Controlling extradural venous congestion is essential to obtain a clear operative field in cases of arteriovenous shunt disease at the craniovertebral junction. The link to the video can be found at: https://youtu.be/fCT69WtAQbo.
\end{abstract}

Conflict of Interest

None declared.

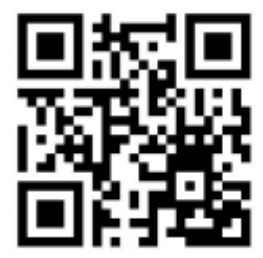

received

February 16, 2019 accepted

August 25, 2019

published online

October 17, 2019

www.thieme.com/skullbasevideos

www.thieme.com/jnlsbvideos

DOI https://doi.org/

10.1055/s-0039-1697981. ISSN 2193-6331. (c) 2019 Georg Thieme Verlag KG Stuttgart · New York
License terms

(c) $(1) \$$ 

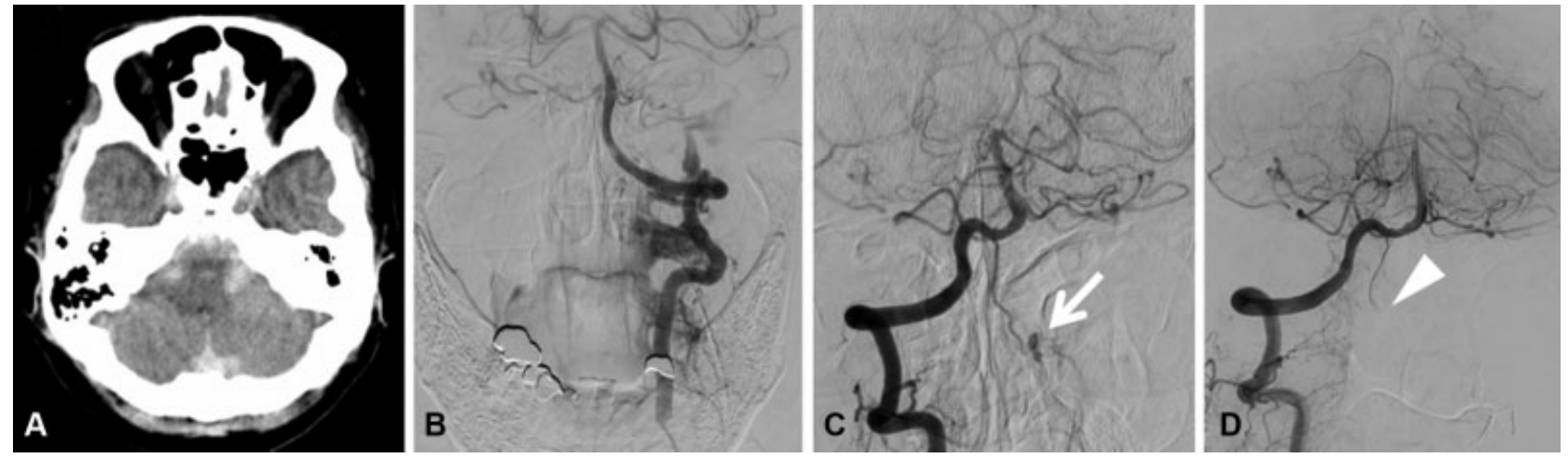

Fig. 1 (A) The initial CT scan showing the subarachnoid hemorrhage. (B) Left vertebral angiogram demonstrating a dural arteriovenous fistula at the craniovertebral junction. (C) Right vertebral angiogram showing an aneurysm (arrow) arising at the aberrant branch of the anterior spinal artery. (D) The postoperative angiogram demonstrating the extirpation of the aneurysm (arrowhead).
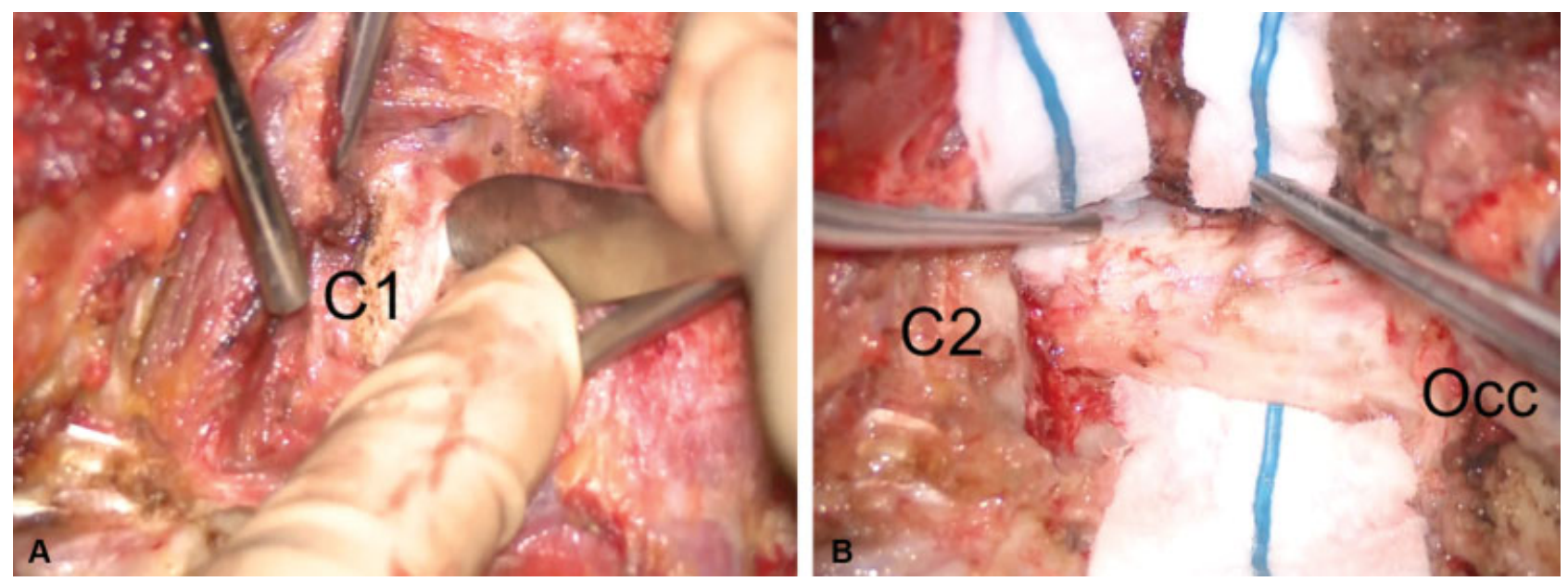

Fig. 2 (A) Intraoperative photograph showing the subperiosteal dissection technique to protect the vertebral artery and minimize the bleeding from the paravertebral plexus. (B) The sufficient posterolateral exposure was obtained with minimal bleeding. C1, C1 lamina; C2, C2 lamina; Occ, occipital bone. 